3-20-1998

\title{
Amplification of High-Order Rainbows of a Cylinder with an Elliptical Cross Section
}

James A. Lock

Cleveland State University, j.lock@csuohio.edu

Charles L. Adler

Bradley R. Stone

Patrick D. Zajak

Follow this and additional works at: https://engagedscholarship.csuohio.edu/sciphysics_facpub

Part of the Physics Commons

How does access to this work benefit you? Let us know!

\section{Publisher's Statement}

This paper was published in Applied Optics and is made available as an electronic reprint with the permission of OSA. The paper can be found at the following URL on the OSA website: http://www.opticsinfobase.org/ao/abstract.cfm?URI=ao-37-9-1527. Systematic or multiple reproduction or distribution to multiple locations via electronic or other means is prohibited and is subject to penalties under law.

\section{Original Citation}

Lock, James A., Charles L. Adler, Bradley R. Stone, and Patrick D. Zajak. "Amplification of High-Order Rainbows of a Cylinder with an Elliptical Cross Section." Applied Optics 37 (1998): 1527-1533.

\section{Repository Citation}

Lock, James A.; Adler, Charles L.; Stone, Bradley R.; and Zajak, Patrick D., "Amplification of High-Order Rainbows of a Cylinder with an Elliptical Cross Section" (1998). Physics Faculty Publications. 74.

https://engagedscholarship.csuohio.edu/sciphysics_facpub/74

This Article is brought to you for free and open access by the Physics Department at EngagedScholarship@CSU. It has been accepted for inclusion in Physics Faculty Publications by an authorized administrator of EngagedScholarship@CSU. For more information, please contact library.es@csuohio.edu. 


\title{
Amplification of high-order rainbows of a cylinder with an elliptical cross section
}

\author{
James A. Lock, Charles L. Adler, Bradley R. Stone, and Patrick D. Zajak
}

\begin{abstract}
The intensity of high-order rainbows for normally incident light and certain rotation angles of a cylinder with an elliptical cross section is greatly amplified with respect to the intensity for a circular crosssectional cylinder. The amplification is due to a number of the internal reflections occurring past the critical angle for total internal reflection, and the effect is especially strong for odd-order rainbows, beginning with the third order. Experimentally, the fourth- and the fifth-order rainbows of a nearly elliptical cross-sectional glass rod were observed and analyzed. (C) 1998 Optical Society of America

OCIS codes: $090.5640,290.0290$
\end{abstract}

\section{Introduction}

The extreme rarity of naked-eye observations of rainbows beyond the second order in rain showers is due in part to the fact that the intensity of high-order rainbows decreases approximately geometrically as a function of the number of internal reflections of light rays in the water droplets. ${ }^{1}$ As a result, the highorder rainbows are lost in the background sky brightness. Likewise, laboratory observations of high-order rainbows of a single liquid droplet are hampered by weak rainbow signals being obscured by the much brighter specularly reflected and transmitted light exiting the droplet in the same direction. ${ }^{2}$ In spite of these difficulties, most of the rainbows to the 24th order, as well as the 32nd-order rainbow, have been observed in the laboratory ${ }^{3}$ by use of bright-light sources, sensitive detectors, and by use of either incident-beam-blocking techniques ${ }^{1,4}$ or off-axis Gaussian beam illumination ${ }^{5}$ to channel as much power as possible into the impact parameter of the rainbow ray and to avoid producing the otherwise dominant specularly reflected and transmitted light.

In this paper we consider the laboratory observation of high-order rainbows of a dielectric cylinder,

J. A. Lock and P. D. Zajak are with the Department of Physics, Cleveland State University, Cleveland, Ohio 44115. C. L. Adler is with the Department of Physics, St. Mary's College of Maryland, St. Mary's City, Maryland 20686. B. R. Stone is with the Department of Electrical Engineering, University of Dayton, Dayton, Ohio 45469.

Received 20 June 1997; revised manuscript received 6 October 1997.

0003-6935/98/091527-07\$15.00/0

(C) 1998 Optical Society of America such as a long glass rod. We show that for normally incident light and certain rotation angles of a cylinder with an elliptical cross section, the intensity of highorder rainbows should be greatly amplified with respect to the intensity of the corresponding rainbows of a circular cross-sectional cylinder. This is because a number of the internal reflections of the rainbow ray occur past the critical angle for total internal reflection. We show that even if the major and minor axes of the cylinder's cross section differ by as little as a few percent, rainbows as high as the fifth order can, under the proper conditions, become as bright as the first-order rainbow.

The body of this paper is organized as follows. In Section 2 we outline the geometric ray theory of highorder rainbows of a cylinder whose cross section is either elliptical or comprises two half-ellipses of different eccentricities smoothly joined together. The twohalf-ellipse model was chosen because the analytical formulas describing the propagation of a ray inside the cylinder are relatively straightforward. In addition, this model closely approximates the nearly elliptical cross section of the glass rod that we previously used to observe low-order rainbows at either normal ${ }^{6}$ or diagonal incidence ${ }^{7}$ as well as interior optical caustics at diagonal incidence. ${ }^{8}$ In this paper we numerically compute both the rainbow angle and the contribution of the Fresnel coefficients to the intensity for rainbows up to the seventh order for an elliptical cross-sectional cylinder. We find that the rainbow intensity should dramatically increase for certain rotation angles of the cylinder. The intensity increase is most dramatic for odd-order rainbows because every second internal reflection is a total internal reflection. In Section 3 we perform an experimental search for high-order rain- 
bows of a nearly elliptical cross-sectional glass rod and report our observations of the fourth- and the fifthorder rainbows. Last, in Section 4 we discuss our results and comment on strategies for observing the third-order rainbow of falling oblate spheroidal water droplets in a rain shower.

\section{Theory of High-Order Rainbow Formation by a Cylinder with a Two-Half-Ellipse Cross Section}

As in Ref. 6, we consider a long homogeneous dielectric cylinder of refractive index $n$, whose symmetry axis coincides with the $z^{\prime}$ axis and whose cross section is given by

$$
\begin{array}{ll}
\frac{x^{\prime 2}}{a^{2}}+\frac{y^{\prime 2}}{b_{1}^{2}}=1 & \text { for } y^{\prime} \geq 0, \\
\frac{x^{\prime 2}}{a^{2}}+\frac{y^{\prime 2}}{b_{2}^{2}}=1 & \text { for } y^{\prime}<0,
\end{array}
$$

where the $x^{\prime} y^{\prime} z^{\prime}$ coordinate system is attached to the cylinder. The cylinder is rotated about the $z=z^{\prime}$ axis through the angle $\xi$ ( $\xi$ is the angle between the $x$ and the $x^{\prime}$ axes) and parallel light rays traveling in the $-y$ direction of the laboratory $x y z$ coordinate system are normally incident upon the cylinder. An incident light ray strikes the illuminated side of the cylinder at the laboratory coordinate $\left(x_{0}, y_{0}\right)$ and is refracted into the cylinder. It then internally reflects at the surface $P-1$ times at the coordinates $\left(x_{p}, y_{p}\right)$ with $1 \leq p \leq P-1$ and finally refracts out of the cylinder at $\left(x_{p}, y_{p}\right)$ with the scattering angle $\theta_{P}(\xi)$. The relative minimum of $\theta_{P}(\xi)$ as a function of $x_{0}$ is denoted by $\theta_{P}{ }^{R}(\xi)$ and is the $(P-1)$ th-order rainbow angle for the cylinder rotation angle $\xi$.

As was described in detail in Ref. 6, the normal to the cylinder surface at $\left(x_{p}, y_{p}\right)$ and the interior ray between the $p$ and the $p+1$ interactions with the surface make the angles $\gamma_{p}$ and $\delta_{p}$, respectively, with the positive $x$ axis. For a cylinder with a two-halfellipse cross section, we have

$$
\tan \gamma_{p}=\frac{\left(b_{j}{ }^{2} / a^{2}\right) \sin ^{2} \xi+\cos ^{2} \xi-x_{p}{ }^{2}}{\left(b_{j}{ }^{2} / a^{2}-1\right) \sin \xi \cos \xi+x_{p} y_{p}}
$$

for the $j=1,2$ half-ellipses. The angles of incidence and transmission of the incoming ray at the point $\left(x_{0}\right.$, $y_{0}$ ) on the cylinder surface are

$$
\begin{aligned}
& \theta_{0}{ }^{i}=\pi / 2-\gamma_{0}, \\
& \theta_{0}{ }^{t}=\arcsin \left[\left(\sin \theta_{0}{ }^{i}\right) / n\right] .
\end{aligned}
$$

The angles $\delta_{p}$ of the interior rays are

$$
\begin{array}{rlrl}
\delta_{0} & =\gamma_{0}+\theta_{0}^{t}, & \\
\delta_{p}=2 \gamma_{p}-\delta_{p-1} & & \text { if } p \geq 1, p=\text { odd, } \\
& =2 \gamma_{p}-\delta_{p-1}-\pi & & \text { if } p \geq 1, p=\text { even, }
\end{array}
$$

and the angle of incidence of the interior ray at $\left(x_{p}\right.$, $\left.y_{p}\right)$ is

$$
\theta_{p}^{i}=\gamma_{p}-\delta_{p-1} .
$$

Last, the scattering angle of the ray exiting the cylinder at $\left(x_{p}, y_{p}\right)$ after $P-1$ internal reflections is

$$
\theta_{P}(\xi)=\begin{array}{ll}
P \pi / 2-\gamma_{P}+\theta_{P}{ }^{t} & \text { if } P=\text { odd }, \\
(P+1) \pi / 2-\gamma_{P}+\theta_{P}{ }^{t} & \text { if } P=\text { even }
\end{array}
$$

where

$$
\theta_{P}{ }^{t}=\arcsin \left(n \sin \theta_{P}{ }^{i}\right) .
$$

As was the case in Ref. 6, at each interaction of a ray with the cylinder surface a decision must be made as to whether the interaction takes place on the $j=1$ half-ellipse or on the $j=2$ half-ellipse. The decision is made by examination of whether the internal ray between the $p$ and the $p+1$ interactions crosses the line that connects the two points on the circumference where the half-ellipses are joined together. Numerically propagating a ray inside the cylinder begins with specifying $\left(x_{p}, y_{p}\right)$, calculating $\gamma_{p}$, then calculating $\delta_{p}$, and then calculating $\left(x_{p+1}, y_{p+1}\right)$ with the expression for $y_{p+1}$ obtained when Eqs. (1) in the laboratory coordinate system are substituted into

$$
\tan \delta_{p}=\left(y_{p+1}-y_{p}\right) /\left(x_{p+1}-x_{p}\right) .
$$

The contribution of the Fresnel coefficients to the intensity of the $P$ rainbow [also called the $(P-1)$ thorder rainbow] for unpolarized incident light is

$$
I_{P}=\frac{1}{2}\left[t_{0}^{\mathrm{TE}}\left(\prod_{p=1}^{P-1} r_{p}^{\mathrm{TE}}\right) t_{P}^{\mathrm{TE}}\right]^{2}+\frac{1}{2}\left[t_{0}^{\mathrm{TM}}\left(\prod_{p=1}^{P-1} r_{p}^{\mathrm{TM}}\right) t_{P}^{\mathrm{TM}}\right]^{2},
$$

where $t^{\mathrm{TE}}, r^{\mathrm{TE}}, t^{\mathrm{TM}}$, and $r^{\mathrm{TM}}$ are the transverse electric (TE) polarization and transverse magnetic (TM) polarization electric-field transmission $(t)$ and reflection $(r)$ Fresnel coefficients evaluated at the angles of incidence and transmission of the rainbow ray at each interaction with the surface. Equation (10) is the dominant contribution to the rainbow intensity in Airy theory for a circular cross-sectional cylinder.9,10 Another contribution to the intensity whose quantitative importance has not been studied here is the dependence of the spreading rate of the local outgoing flux tube on the curvature of the cylinder at the ray interaction points. This flux tube effect for scattering by spheres and spheroids is described in Refs. 11 and 12.

The ray-tracing procedure of Eqs. (2)-(9) was used to compute numerically the rainbow angle $\theta_{P}{ }^{R}(\xi)$ as a function of $\xi$ in $5^{\circ}$ increments for $2 \leq P \leq 8, n=1.474$, and for various values of $b / a$ for an elliptical crosssectional cylinder with $b \equiv b_{1}=b_{2} \neq a$. Figure 1 shows representative results for the $P=6$ rainbow with $b / a=1.0,0.987,0.975,0.963$, and 0.950. The graphs in Fig. 1 are given only for $0^{\circ} \leq \xi \leq 180^{\circ}$ because, for an elliptical cross-sectional cylinder, $\theta_{P}{ }^{R}(\xi)$ has $180^{\circ}$ rotational symmetry and the results for $180^{\circ}$ $\leq \xi \leq 360^{\circ}$ repeat the $0^{\circ} \leq \xi \leq 180^{\circ}$ results. By 


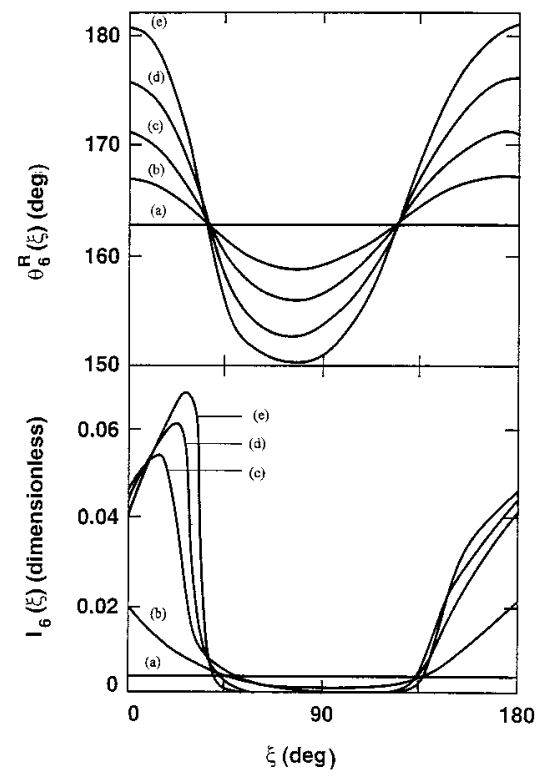

Fig. 1. Rainbow angle $\theta_{6}{ }^{R}$ and the rainbow Fresnel coefficient factor $I_{6}$ as defined in Eq. (10) for the $P=6$ rainbow of an elliptical-cross-section cylinder as a function of the cylinder rotation angle $\xi$ for (a) $b / a=1.0$, (b) $b / a=0.987$, (c) $b / a=0.975$, (d) $b / a=0.963$, (e) $b / a=0.950$.

comparison, the $P=6$ rainbow angle of a circular cross-sectional cylinder with $n=1.474$ and $b / a=1.0$ is $163.09^{\circ}$ for all $\xi$ and $I_{6}=0.0043$. For an elliptical cross-sectional cylinder with $b / a<1$, the rainbow angle increasingly deviates from its $P=6$ circular crosssectional value as $b / a$ decreases. But more significantly, $I_{6}$ quickly grows to more than an order of magnitude larger than the circular cross-sectional value in certain $\xi$ intervals whereas it shrinks to more than an order of magnitude smaller than the circular cross-sectional value in other $\xi$ intervals. The reason for this is apparent in Fig. 2, in which each of the individual TE Fresnel coefficients of the $P=6$ rainbow ray is graphed as a function of $\xi$. The region of large $I_{6}$ amplification corresponds to the $p=1,3,5$ reflections becoming total internal reflections, and the region of minimum $I_{6}$ corresponds to the minimum values of the $p=1,3,5$ reflection Fresnel coefficients. The varying curvature of the elliptical cross section can thus be exploited to create situations in which total internal reflection occurs, and, as a result, the rainbow intensity dramatically increases. For the sake of completeness, it should be noted that a small evanescent wave leakage occurs by means of tunneling at these total internal reflections, ${ }^{13}$ rendering the reflected percentage there to not be strictly $100 \%$.

The trends observed for the $P=6$ rainbow occur for other high-order rainbows as well. In Tables 1 and 2 we list the maximum value of $I_{p}$, the value of the cylinder rotation angle $\xi$ at which it occurs (to the nearest $5^{\circ}$ increment), and the Fresnel coefficient amplification ratio with respect to a circular crosssectional cylinder for the first seven rainbows and $b / a=0.975,0.950$. These tables exhibit the following trends. Although the first- and the second-order rainbow intensities are likely to be only minimally

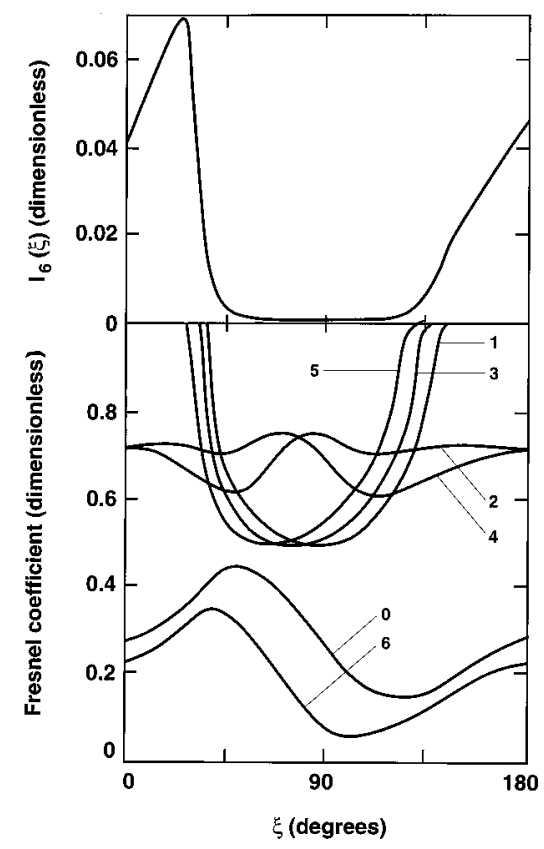

Fig. 2. Rainbow Fresnel coefficient factor as defined in Eq. (10) and the values of the individual TE Fresnel coefficients for the $P=$ 6 rainbow of an elliptical-cross-section cylinder for the $0 \leq p \leq 6$ interactions of the rainbow ray with the cylinder surface. The $p=$ 0 curve is the initial transmission coefficient, the $1 \leq p \leq 5$ curves are the internal reflection coefficients, and the $p=6$ curve is the final transmission coefficient minus one.

amplified for a cylinder with a slightly elliptical cross section, based on the Fresnel coefficient factors, the intensity amplification of high-order rainbows in certain narrow intervals of $\xi$ should be substantial, especially for even- $P$ (i.e., odd-order) rainbows. It is also significant that the maximum amplification occurs at nearly the same value of $\xi, \sim 20^{\circ}$ to $25^{\circ}$ for even $P$ and $\sim 30^{\circ}$ to $35^{\circ}$ for odd $P$ when $b / a=0.95$. For $b / a=0.95$, the $P=4$ rainbow is at $\theta \approx 0^{\circ}$ and is predicted to have roughly twice the peak intensity of the first-order rainbow for $n=1.474$. The $P=6$ rainbow is at $\theta \approx 180^{\circ}$ and is predicted to have roughly the same peak intensity as the first-order rainbow, assuming that the flux tube spreading factors play a small role. Such should be the case here, as the local radius of curvature varies by only $-10 \%$ to $+5 \%$ around the cylinder perimeter for $b / a=0.95$.

Table 1. Maximum Fresnel Coefficient Contribution to the Rainbow Intensity for an Elliptical Cross-Sectional Cylinder, the Cylinder Rotation Angle $\xi$ at which it occurs, and the Fresnel Coefficient Amplification Ratio with respect to a Circular Cross-Sectional Cylinder for the $2 \leq$ $P \leq 8$ Rainbows and $b / a=0.975$

\begin{tabular}{lccc}
\hline$P$ & $I_{P}^{\max }$ & $\xi(\mathrm{deg})$ & $I_{P}{ }^{\max } / I_{P}{ }^{\text {circle }}$ \\
\hline 2 & 0.0520 & 20 & 1.16 \\
3 & 0.0196 & 35 & 1.10 \\
4 & 0.0315 & 5 & 3.21 \\
5 & 0.0104 & 20 & 1.69 \\
6 & 0.0554 & 15 & 12.98 \\
7 & 0.0172 & 35 & 5.47 \\
8 & 0.0363 & 15 & 15.23 \\
\hline
\end{tabular}


Table 2. Maximum Fresnel Coefficient Contribution to the Rainbow Intensity for an Elliptical Cross-Sectional Cylinder, the Cylinder Rotation Angle $\xi$ at which it occurs, and the Fresnel Coefficient Amplification Ratio with respect to a Circular Cross-Sectional Cylinder for the $2 \leq P \leq 8$ Rainbows and $b / a=0.950$

\begin{tabular}{cccc}
\hline$P$ & $I_{P}^{\max }$ & $\xi(\mathrm{deg})$ & $I_{P}^{\max } / I_{P}{ }^{\text {circle }}$ \\
\hline 2 & 0.0621 & 20 & 1.39 \\
3 & 0.0219 & 30 & 1.22 \\
4 & 0.1298 & 25 & 13.22 \\
5 & 0.0178 & 30 & 2.89 \\
6 & 0.0688 & 25 & 16.11 \\
7 & 0.0405 & 35 & 12.89 \\
8 & 0.0386 & 20 & 16.23 \\
\hline
\end{tabular}

For each of the maximum Fresnel coefficient amplifications in Table 2, the individual Fresnel coefficients were examined, and their behavior is summarized in Fig. 3. The $P=4,6,8, \ldots$, rainbow peak intensities should be greatly amplified because every second reflection (i.e., $p=1,3,5,7 \cdots$ ) is a total internal reflection. This also explains why the $\xi$ interval for maximum amplification is nearly the same for each even- $P$ rainbow. The impact parameter of a highorder rainbow ray is near the edge of the cylinder, and four internal reflections for $n=1.474$ brings the rainbow ray back to the vicinity of its starting point. Thus every second interaction occurs on opposite sides of the cylinder within the low-curvature regions on the circumference where total internal reflection occurs. The intensity amplification mechanism is modified somewhat for odd- $P$ rainbows because the rainbow ray must be able to exit the cylinder at the last odd- $P$ interaction and not be totally internally reflected there. This leads to the maximum Fresnel coefficient amplification occurring at a somewhat different value of $\xi$ at which the elliptical cross section has a different orientation and at which the last interaction of the rainbow ray with the surface no longer is within one of the low-curvature total internal reflection regions on the circumference. As a result, for odd- $P$ rainbows there are relatively fewer total internal reflections and a correspondingly lower peak amplification ratio.

From an experimental point of view, although rainbow intensity amplification for an elliptical crosssectional cylinder due to total internal reflections is both novel and interesting, glass rods are seldom manufactured with an exactly elliptical cross section. They possess small deviations from ellipticity, such as the glass rod that we have previously used, ${ }^{6}$ which had an average radius of $a=8.05 \mathrm{~mm}$ and whose cross section was reasonably well described by the two-halfellipse model with $b_{1} / a=0.950$ and $b_{2} / a=0.976$. A question of experimental interest is whether the large amplification ratios obtained for an elliptical cross section persist if the cross section contains small deviations from ellipticity. In Fig. 4 we compare the rainbow angle and $I_{p}$ as functions of $\xi$ for the $P=6$ rainbow of our glass rod predicted by the two-halfellipse model with those of an elliptical cross-sectional cylinder with an eccentricity of $b / a=0.963$, the average of $b_{1} / a$ and $b_{2} / a$. The figure shows that although
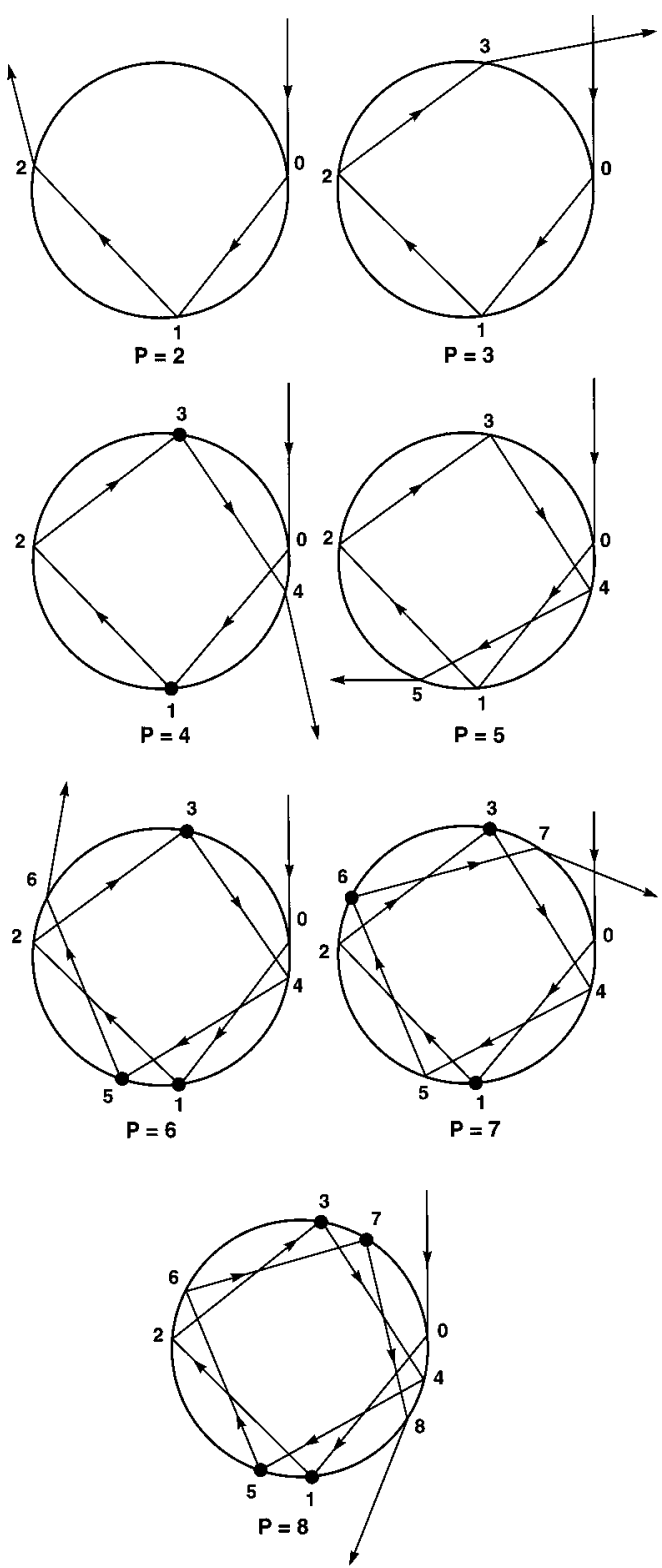

Fig. 3. Ray path of the rainbow ray for $2 \leq P \leq 8$ and $n=1.474$ at the cylinder rotation angle corresponding to the maximum rainbow Fresnel coefficient amplification given in Table 2. The large filled circles represent total internal reflection.

small deviations from ellipticity cause noticeable differences in both the rainbow angle and $I_{p}$ (i.e., the first Fresnel coefficient amplification maximum in Fig. 4 is broadened and further increased whereas the second maximum is narrowed and decreased), the basic phenomenon of rainbow intensity amplification persists. Small deviations from ellipticity change the rainbow ray path inside the cylinder and the interaction points of the ray with the surface. But the changes apparently come close to averaging each other out rather than producing a systematic shift that could cause substantial differences in the rainbow angle and a dramatic decrease in the rainbow Fresnel coefficient amplification ratio. The stability of the Fresnel coefficient amplification on small perturbations from 


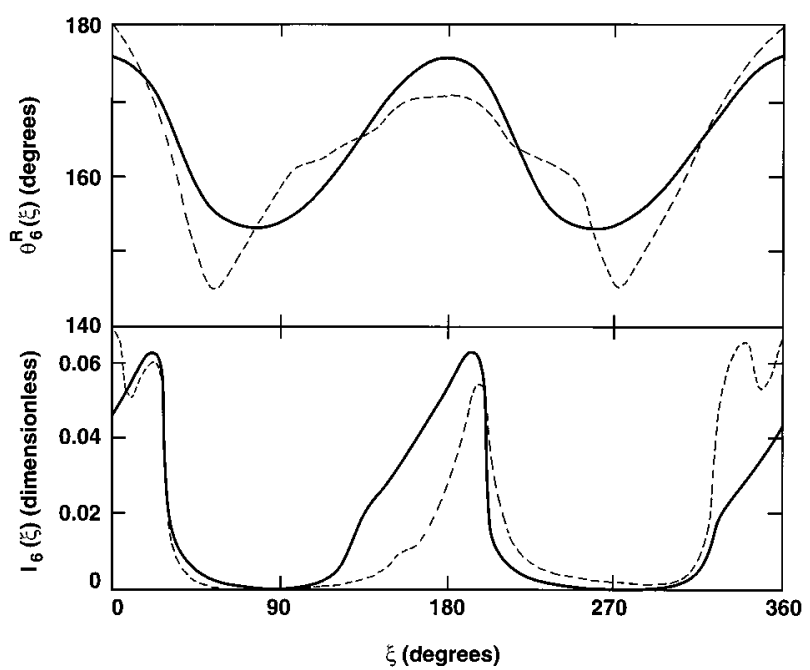

Fig. 4. Rainbow angle $\theta_{6}{ }^{R}$ and the rainbow Fresnel coefficient factor $I_{6}$ as defined in Eq. (10) for the $P=6$ rainbow as a function of the cylinder rotation angle $\xi$ for an elliptical cross section with $b / a=0.963$ (solid curve) and a two-half-ellipse cross section with $b_{1} / a=0.950$ and $b_{2} / a=0.976$ (dashed curve).

ellipticity provides encouragement for experimentally searching for the high-order rainbows of a nearly elliptical cross-sectional glass rod.

\section{Experiment}

Our experimental apparatus has been described elsewhere. ${ }^{6-8}$ Briefly, the glass rod was placed on a calibrated rotation stage and was normally illuminated with the expanded beam of a $15-\mathrm{mW} \mathrm{He}-\mathrm{Ne}$ laser. Previous observations of the $P=2,3$ rainbows permitted the determination of the rod's refractive index, average ellipticity, and ellipticity difference by use of the two-half-ellipse crosssectional model. As mentioned in Section 2, the $P=$ 4 rainbow should occur near $\theta \approx 0^{\circ}$ and is lost in the bright transmitted light. The $P=8$ rainbow should also occur far enough into the forward hemisphere (i.e., $\theta \approx 15^{\circ}$ ) so as to be obscured by the transmitted light. Thus the most promising candidates for observation are the $P=5,6,7$ rainbows, which should occur at the scattering angles $\theta \approx 90^{\circ}, \theta \approx 180^{\circ}$, and $\theta \approx 75^{\circ}$, respectively, when the rod is rotated into the proper $\xi$ interval for maximal amplification.

Despite their expected large peak brightness, these high-order rainbows are elusive, however, as their intensities should be quite small for most other values of $\xi$. As the rod is rotated they should quickly flash into visibility, and then a few degrees in $\xi$ later they should equally quickly fade away. The situation is further complicated by the fact that the experimental observations of the $P=2,3$ rainbows in Ref. 6 were fitted well, but not perfectly, by the two-half-ellipse cross-sectional model because the shape of our rod's cross section is in actuality somewhat more complicated. As a result, the two-half-ellipse model provides only a rough estimate of where the high-order rainbows should occur.

The $P=5$ rainbow was first seen during our previous study of the $P=3$ rainbow when the two rainbows

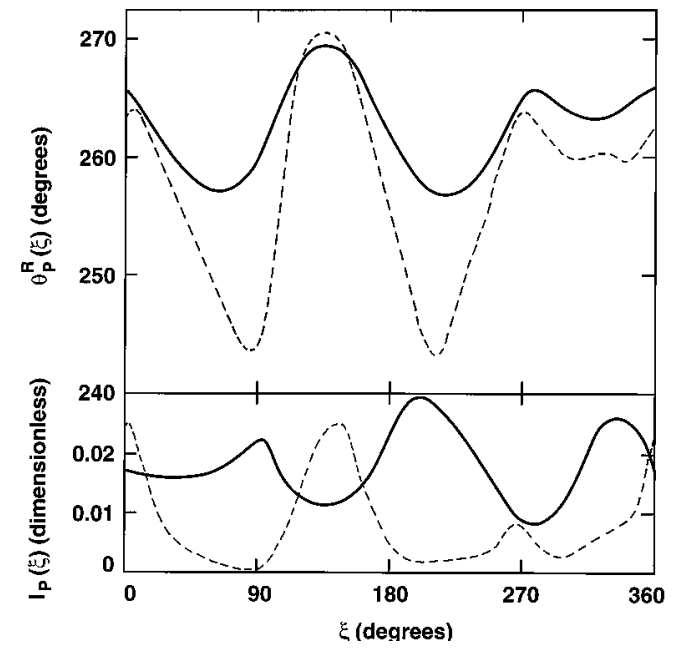

Fig. 5. Rainbow angle $\theta_{P}{ }^{R}$ and the rainbow Fresnel coefficient factor $I_{P}$ as defined in Eq. (10) for the $P=3$ (solid curve) and $P=$ 5 (dashed curve) rainbows as a function of the cylinder rotation angle $\xi$ for a two-half-ellipse cross section with $b_{1} / a=0.950$ and $b_{2} / a=0.976$. The two rainbows are produced by rays incident at opposite sides of the cylinder.

occurred at nearly the same scattering angle. In Fig. 5 we show the predicted $P=3,5$ rainbow angles and Fresnel coefficient amplification factors of Eq. (10) for our glass rod in the two-half-ellipse cross-sectional model. The $P=3$ rainbow intensity is predicted to vary by a factor of $\sim 2$, ignoring variations in the spreading rate of the outgoing flux tube. Such a variation was qualitatively observed in the laboratory, and the minimum $P=3$ rainbow intensity (i.e., $I_{p} \approx 0.01$ in Fig. 5) roughly coincided with our visual observation threshold. The $P=5$ rainbow intensity has predicted sharp enhancements when it is in the vicinity of the $P=3$ rainbow at the $P=3$ closest approach to the forward direction. It was at these two locations $(\xi \approx$ $0^{\circ}$ and $\xi \approx 140^{\circ}$ corresponding to $\theta_{3}{ }^{R} \approx 270^{\circ}$ ) that we observed the $P=5$ rainbow within a few degrees of the $P=3$ rainbow. As expected, it quickly flashed into existence, moved across the viewing screen more rapidly than did the $P=3$ rainbow, and then equally quickly faded from view as $\xi$ was varied by $\sim 5^{\circ}$ in one case and by $\sim 10^{\circ}$ in the other. By using the beamblocking technique, we verified that the rainbow rays of the $P=3,5$ rainbows originated on opposite sides of the cylinder, as expected from Fig. 3. Also as expected, the observed supernumerary patterns of the two rainbows faced each other, with the principal peaks being on opposite ends of the pattern. The light intensity in the vicinity of the $P=3,5$ rainbows was measured with a photodiode mounted on a fixed track and advanced by a stepper motor, as was described in Ref. 6. The resulting intensities of the two rainbows are given in Figs. 6 and 7. As expected, the separation of the $P=5$ supernumeraries is wider than that of the $P=3$ supernumeraries. But their intensity shows significant deviations from the square of an Airy function. This was also noted in Ref. 6 for the $P=2$ rainbow and may be due to small local inhomogeneities in the rod's glass. The $P=5$ rainbow visually 


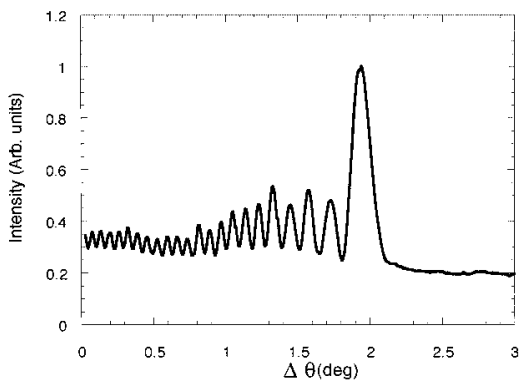

Fig. 6. Experimental light intensity in the vicinity of the $P=3$ rainbow at $\theta_{3}{ }^{R} \approx$ $270^{\circ}$. The distance from the glass rod to the detector was $49.5 \mathrm{~cm}$.

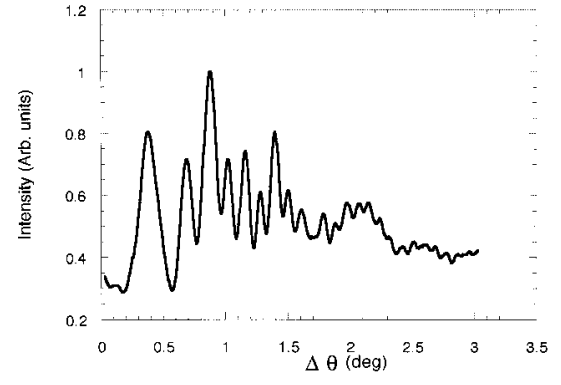

Fig. 7. Experimental light intensity in the vicinity of the $P=5$ rainbow at $\theta_{5}{ }^{R} \approx 275^{\circ}$. The distance from the glass rod to the detector was $49.5 \mathrm{~cm}$.

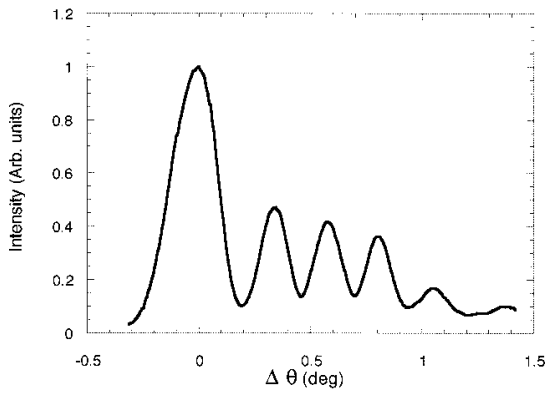

Fig. 8. Experimental light intensity in the vicinity of the $P=6$ rainbow at $\theta_{6}{ }^{R} \approx 175^{\circ}$. The distance from the glass rod to the detector was $73.5 \mathrm{~cm}$. appeared more like the square of an Airy function than the intensity scan of Fig. 7 indicates. The pattern as viewed by the authors consisted of the principal rainbow peak flanked on one side by eight clear supernumeraries, one or two of which appeared somewhat brighter than the principal peak. This is evident in Fig. 7 along with a relatively large background noise level that was not visible to the naked eye.

Next, with two viewing screens set so that the expanded incident beam passed through a $20-\mathrm{mm}$ gap between them, the rod was rotated until the $P=6$ rainbow was observed on one of the viewing screens at a scattering angle a few degrees away from $180^{\circ}$. Again, as $\xi$ was varied, it quickly flashed into existence, and after another $\sim 3^{\circ}$ of rotation it equally quickly faded from view. Once the rainbow was found, the viewing screens were brought closer together until the competing specularly reflected light was eliminated. We verified by the beam-blocking technique that the $P=6$ rainbow was due to light incident at the edge of the cylinder. The $P=2$ rainbow could also be seen on one of the viewing screens, and the $P=6$ principal maximum visually appeared to be of comparable brightness with that of the $P=2$ rainbow, whereas the $P=6$ supernumeraries were noticeably dimmer than those of $P=2$. In comparison, the principal peak of the $P=3$ rainbow was much dimmer than the $P=2$ principal peak. The intensity in the vicinity of the $P=6$ rainbow was then measured with the photodiode driven by the stepper motor and is shown in Fig. 8 . We did not attempt to locate the $P=7$ rainbow.

The angular separations of the respective supernumeraries of the $P=3,5,6$ rainbows of a circular crosssectional cylinder for $n=1.474$ are in the ratio 1:1.81: 2.20 according to Airy theory. ${ }^{9,10}$ Analysis of the first four supernumerary maxima and minima of Figs. 6-8 gives the ratio 1:1.39:1.60. Although the trend of increasingly wide supernumeraries as $P$ is increased is apparent in Figs. 6-8, the numerical values of the ratios are incorrect because of the supernumerary patterns of the $P=2,3$ rainbows growing and shrinking by a factor of $\sim 2$ as the rod was rotated through a complete revolution, as was mentioned in Ref. 6. The reason for this is most likely a combination of small local inhomogeneities in the glass and variations in the spreading rate of the outgoing flux tube.

\section{Discussion}

Searching for the high-order rainbows of an ellipticalcross-section or nearly elliptical cross-sectional cylinder possesses both advantages and disadvantages relative to searching for the high-order rainbows of a circular cross-sectional cylinder. The advantage is that the varying curvature of the elliptical cross section provides an opportunity for a number of the internal reflections to become total, thus rendering the high-order rainbows much brighter than their circular cross-sectional counterparts. The disadvantage is that, because the brightening occurs over a small range of the cylinder rotation angle $\xi$, it is difficult to predict exactly when in $\xi$ and where in $\theta$ the bright high-order rainbows will occur if the cross section is not exactly elliptical. The results of Table 2 and Fig. 3 illustrate that the total internal reflections occur in the regions where the surface curvature is smaller than average. But for a rod whose ellipticity is of the order of only a few percent, these low-curvature regions are difficult to locate visually.

In spite of this disadvantage, we observed the $P=5$, 6 rainbows of a nearly elliptical cross-sectional glass rod illuminated by the expanded beam of a low-power He-Ne laser. Had the direct unexpanded beam been incident at the edge of the cylinder, we no doubt would have been able to observe additional high-order rainbows because of increased laser power being channeled into the rainbow ray impact parameter. Indeed, when we placed the glass rod in a tank of water into which a few drops of milk had been added and illuminated the rod's edge with the unexpanded laser beam, we easily saw the first ten rainbows in the light scattered by the suspended milk particles. Each rainbow ray was flanked on one side by a small bundle of supernumerary rays, and each group of rays left the cylinder circumference nearly tangentially at $\sim 50^{\circ}$ intervals. A few of the rainbows visually appeared dimmer than the neighboring higher- and lower- $P$ rainbows because of small Fresnel reflection coefficients for the particular rod rotation angle used.

Variants of the total internal reflection mechanism for high-order rainbow amplification have been exploited previously. In order to amplify the rainbowenhanced forward glory, D'Amico et al. reflectively 
coated their fused-silica spheres at the locations where internal reflections were expected to occur. ${ }^{14}$ Similarly, we previously observed the $P \leq 6$ internal caustics of the glass rod illuminated by an expanded laser beam at diagonal incidence and at $\xi=90^{\circ}$. In that situation as well, the internal reflection coefficients attained much larger values at diagonal incidence than at normal incidence. ${ }^{8}$ The relative refractive index of the glass rod in water for the qualitative observations described above is 1.106. For a low refractive index, each rainbow ray is incident comparatively nearer the edge of the cylinder, the internal reflections occur nearer the critical angle for total internal reflection, and high-order rainbows become dimmer at a progressively slower rate, thus permitting easier observation of high-order rainbows.

Last, a complementary phenomenon observed in asymmetrical optical cavities has attracted much attention recently. ${ }^{15,16}$ In a circular cross-sectional cavity, morphology-dependent resonances (MDR's) can attain long lifetimes because of successive interactions with the cavity surface occurring beyond the critical angle for total internal reflection. When the cavity is deformed, however, the interior MDR ray is occasionally incident upon the cavity surface at an angle less than the critical angle, leading to refractive intensity losses and a resulting decrease in the MDR lifetime. This effect has been called chaotic light because of the nature of the MDR ray path inside the cavity. ${ }^{17}$ In the analysis of this phenomenon, the cavity cross section has been modeled as a small quadrupole deformation of a circular shape rather than as an ellipse, or two half-ellipses, as is done here.

The results of Tables 1 and 2 are virtually unchanged if the refractive index 1.333 corresponding to water is used, rather than 1.474 for glass. The intensity of the $P=2,3$ rainbows varies only weakly as a function of $\xi$. The maximum amplification of the $P=4,6,8, \ldots$, rainbows is large and occurs for $\xi \approx 20^{\circ}$, whereas the maximum amplification for $P=5,7, \ldots$, is somewhat smaller and occurs for $\xi \approx 0^{\circ}$. The interval in $\xi$ of maximum amplification becomes narrower as $P$ increases. Again, because four internal reflections of a rainbow ray within the elliptical cross section bring the ray back to roughly its starting point for $n=1.333$, the high value of the maximum amplification ratio for even $P$ (i.e., odd-order rainbows) is due to every second reflection being a total internal reflection.

Finally, we consider atmospheric rainbows of falling oblate spheroidal water droplets in a rain shower when the light rays are confined to the vertical plane in which the droplets' cross section is elliptical. The weak variation in the brightness of the $P=2,3$ rainbows as a function of the direction of incidence of light rays from the Sun is sufficiently small so as to not affect the observation of the first two atmospheric rainbows. The region of large amplification of the $P=4$ (i.e., third-order) rainbow intensity is relatively wide in $\xi$ and occurs for high solar elevations, i.e., $50^{\circ}$ or more. When the droplet is large, e.g., $a=0.7 \mathrm{~mm}$ corresponds ${ }^{18}$ to $b / a=0.97$, the maximum Fresnel coefficient amplification ratio is roughly 4. This suggests that a possible opportunity for observing the rare third-order rainbow should occur for ray paths in the vertical plane of relatively large and monodisperse raindrops at high solar elevations with a suitably dark background sky above the Sun. However, during the simultaneous observation of the first-, second-, and thirdorder rainbows, as described in Ref. 19, the Sun was at a relatively low angle. This would indicate that the water droplets involved either were smaller so that their oblate spheroidal flattening was minimal or that the base of the third-order rainbow, produced by light confined to the droplets' circular horizontal cross section, was observed.

\section{References}

1. J. D. Walker, "Multiple rainbows from single drops of water and other liquids," Am. J. Phys. 44, 421-433 (1976).

2. J.A. Lock, "Theory of the observations made of high-order rainbows from a single water droplet," Appl. Opt. 26, 5291-5298 (1987).

3. C. W. Chan and W. K. Lee, "Measurement of a liquid refractive index by using high-order rainbows," J. Opt. Soc. Am. B 13, 532-535 (1996)

4. J. Walker, "How to create and observe a dozen rainbows in a single drop of water," Sci. Am. 237(1), 138-144 (1977).

5. J. A. Lock, "Contribution of high-order rainbows to the scattering of a Gaussian laser beam by a spherical particle," J. Opt. Soc. Am. A 10, 693-706 (1993).

6. C. L. Adler, J. A. Lock, and B. R. Stone, "Rainbow scattering by a cylinder with a nearly elliptical cross section," Appl. Opt. 37, 1540-1550 (1998).

7. J. A. Lock and C. L. Adler, "Debye series analysis of the firstorder rainbow produced in scattering of a diagonally incident plane wave by a circular cylinder," J. Opt. Soc. Am. A 14, 1316-1328 (1997).

8. C. L. Adler, J. A. Lock, B. R. Stone, and C. J. Garcia, "Highorder interior caustics produced in scattering of a diagonally incident plane wave by a circular cylinder," J. Opt. Soc. Am. A 14, 1305-1315 (1997).

9. R. T. Wang and H. C. van de Hulst, "Rainbows: Mie computations and the Airy theory," Appl. Opt. 30, 106-117 (1991).

10. E. A. Hovenac and J. A. Lock, "Assessing the contributions of surface waves and complex rays to far-field Mie scattering by use of the Debye series," J. Opt. Soc. Am. A 9, 781-795 (1992).

11. P. L. Marston, "Geometrical and catastrophe methods in scattering," Phys. Acoust. 21, 1-234 (1992).

12. J. A. Lock, "Ray scattering by an arbitrarily oriented spheroid. I. Diffraction and specular reflection,” Appl. Opt. 35, 500-514 (1996).

13. H. M. Nussenzveig, "High-frequency scattering by a transparent sphere. I. Direct reflection and transmission," J. Math. Phys. 10, 82-124 (1969).

14. J. J. D’Amico, M. D. Knudson, and D. S. Langley, "Rainbowenhanced forward glory from fused-silica spheres," Appl. Opt. 33, 4672-4676 (1994).

15. J. U. Nöckel, A. D. Stone, and R. K. Chang, " $Q$ spoiling and directionality in deformed ring cavities," Opt. Lett. 19, 16931695 (1994).

16. J. U. Nöckel and A. D. Stone, "Ray and wave chaos in asymmetric resonant optical cavities," Nature (London) 385, 45-47 (1997).

17. J. U. Nöckel and A. D. Stone, "Chaotic light: a theory of asymmetric resonant cavities," in Optical Processes in Microcavities, R. K. Chang and A. J. Campillo, eds. (World Scientific, Singapore, 1996), pp. 389-426.

18. H. R. Pruppacher and R. L. Pitter, "A semi-empirical determination of the shape of cloud and rain drops," J. Atmos. Sci. 28, 86-94 (1971).

19. D. E. Pedgley, “A tertiary rainbow," Weather 41, 401 (1986). 